\title{
Impact Of A Specifically Designed Antenatal Class For First-Time Fathers On Their Experience Of Childbirth
}

Isabelle Cornet

University of Applied Sciences and Arts

Begoña Martinez de Tejada

University Hospitals of Geneva

Marie-Julia Guittier ( $\square$ marie-julia.guittier@hesge.ch )

University Hospitals of Geneva https://orcid.org/0000-0003-4894-8362

\section{Research Article}

Keywords: fathers, paternity, childbirth, experience, life changing events, questionnaire

Posted Date: February 17th, 2022

DOI: https://doi.org/10.21203/rs.3.rs-1335707/v1

License: (c) (i) This work is licensed under a Creative Commons Attribution 4.0 International License.

Read Full License 


\section{Abstract}

Purpose: Most fathers describe the experience of witnessing childbirth as very positive, but some feel unsupported and excluded. Offering specific antenatal classes for fathers could improve their experience. This study compares the childbirth experience of first-time fathers who attended specific antenatal classes for men (intervention group) with those who did not (non-intervention group).

Methods: We performed a comparative study at a tertiary maternity hospital in Switzerland using FirstTime Father Questionnaire (FTFQ) one month after childbirth. We assessed the satisfaction of the specific antenatal classes with a questionnaire with open and closed questions.

Results: A total of 121 fathers for the non-intervention group and 52 for the intervention group completed the FTFQ. The score of two of the three dimensions of the FTFQ were significantly better: the professional support was reduced from $1.98(S D=0.52)$ in the non-intervention group to $1.69(S D=0.55)$ in the intervention group, $p=0.001$; and the prenatal preparation from $1.70(S D=0.57)$ to $1.48(S D=0.51)$ respectively, $p=0.019$. In the intervention group, $75 \%$ of fathers-to-be felt better prepared for the childbirth and $95 \%$ enjoyed the classes being for men only. The qualitative analysis of open questions showed positive experiences.

Conclusions: Attending specific antenatal classes for first-time fathers improves the experience of childbirth. Their experience reported in terms of sharing and solidarity seems to be meaningful. Offering fathers-to-be a specifically dedicated space for expression is a way to support their transition to fatherhood.

\section{Introduction}

In Western society, societal expectations of fathers for childbirth have changed significantly in recent decades [1]. Men have become more involved during pregnancy, and particularly during childbirth. Nowadays, they are present in the childbirth units to support their partner, but also to discover their child as soon as possible. Studies have suggested that enhancing fathers' involvement during pregnancy and childbirth might prevent domestic abuse [2] and positively affect the father-infant and partner relationships [3]. In its last recommendations on health promotion interventions for maternal and newborn health, the World Health Organization strongly recommends promoting men's involvement during pregnancy, childbirth and after birth [4]. The objectives are to facilitate and support the improvement of woman's self-care, home care practices for the woman and newborn, and the use of skilled care during pregnancy, childbirth, and the postnatal period. Men recognize that birth is a unique event that may be potentially challenging and requires a level of preparation $[5,6]$.

Indeed, even if most of fathers describe the experience of witnessing childbirth as very positive and feelings of pride, happiness and excitement accompany it [7], some feel unsupported during the process of pregnancy, childbirth and fatherhood $[8,9]$. Often their needs and emotions are ignored, and fathers may feel isolated and excluded during this transition to fatherhood [10,11]. Around $10 \%$ may suffer from 
prenatal or postnatal depression, and 1-6\% may experience post-traumatic stress disorder $[12,13]$. Fathers expecting their first baby seem to be more vulnerable $[14,15]$.

Given the similarities between mothers' and fathers' birth experiences and wellbeing, the study of Hugues et al. highlighted the need for partner-inclusive intervention strategies [16]. To offer a specific space for fathers-to-be to listen and express themselves supports the fatherhood transition through allowing them to develop their social networks and to work through challenging topics, such as gender equality in parenting $[17,18]$. Such groups can create time and space for discussions on all aspects of fatherhood that new fathers find important. Barriers to accessing support included lack of tailored information resources and acknowledgment from health professionals [10]. Better preparation for fatherhood could facilitate better experiences for new fathers and contribute to better adjustments and psychological wellbeing in new fathers $[10,19]$.

The overall aim of this study was to examine the impact of a specifically designed antenatal class for first-time fathers on their experience of childbirth.

\section{Methods}

\section{Design}

We performed an observational comparative study at the maternity unit of the Geneva university hospitals, Switzerland, comparing the experience of childbirth for two groups of first-time fathers: one attending specifically designed prenatal classes (intervention group) and another group not (nointervention group).

\section{Participants and Settings}

Data from first-time fathers who did not attend any specifically designed antenatal class were collected from 2014 to 2015 as part the cross-cultural validation of the FTFQ into French [20]. Data from first-time fathers who participated in an antenatal class were collected from 2019 to 2020 . All participants were invited to participate during this period.

For both groups, the inclusion criteria were first-time fathers, over 18 years old and able to read and write in French.

This study was carried out in the Geneva university hospitals. Its maternity unit is a tertiary referral center and routinely manages high-risk pregnancies, with more than 4,000 births per year. Midwives are responsible for physiological care, and work in collaboration with obstetricians when outside of their skills.

\section{Intervention}

Antenatal classes for fathers were presented as a space for exchange based on an interactive card game between fathers-to-be, a male midwife and another male group facilitator. Three midwives (Busquest, 
Delieutraz-Marchand et Guittier) created the card game. We decided on having a maximum of ten participants per meeting to encourage interactions. Each father had nine cards in front of him: eight themed cards (e.g., "I don't recognize my wife anymore," "My wife is breastfeeding, what good am I?"), and a white card where he could write what he would like to discuss. They choose the cards that spoke to them and gave them to the facilitators. The group chose the subjects, who were thus treated anonymously. These antenatal classes lasted two hours. At the end of the session, a proposal to exchange email addresses and/or cell phone numbers between participants was offered to encourage creating social support. For this pilot period (2019-2020), the courses were free of charge for the participants. All participants received a questionnaire to assess the satisfaction of these specific antenatal classes.

\section{Recruitment and data-collection procedures}

For the non-intervention group included in 2014-2015, the research team approached men during the early post-partum period when they came to visit the mother and baby during their stay in the maternity units. After checking the fathers' inclusion criteria, the research assistants provided information and collected oral consent to participate.

For the intervention group included in 2019-2020, participants in the antenatal classes for father-to-be were informed of the study and invited to participate via an informed form at the end of the antenatal class. If they wished to participate, they noted their contact information and the name of their partner so that we could monitor the due date.

For both groups, depending on their communication preferences, the men participants received the FTFQ via email or mail one month after the birth, in accordance with the deadline the authors of the original questionnaire chose [21].

For the whole study population, two reminders were sent, if necessary, up to six weeks post-partum. Participants could withdraw from the study at any time. Participants who received the questionnaire by mail were allowed not to send it back. Participants who received the questionnaire by email had the option to click on a link that would remove them immediately from this study's mailing list.

Most participants (96\%) wished to complete the questionnaire online. We used the LimeSurvey software package [https://www.limesurvey.org], which is an online survey program commonly used to collect data for scientific research.

\section{Measures}

The questionnaire the participants received involves general background questions, pregnancy and birthrelated questions as well as the FTFQ.

The general background questions involved variables such as their age, their country of birth and their level of education. The pregnancy and birth questions involved variables about the modality of antenatal 
education [apart from specific prenatal classes], the onset of labor, the use of epidural in labor and the mode of birth.

Three questions assessed with a Visual Analog Scale (VAS) were also asked about how useful participants felt during labor and birth, as well as how they felt about the whole birth experience. They could give a rating between 1 and 10, with 1 being the worst experience they could have and 10 the best. An average score was calculated for each of these three questions.

The FTFQ was cross-culturally validated into French in 2016 [20, 22]. The initial Swedish questionnaire consisted of 21 items. The French version involves 19 items divided in three dimensions: professional support (items $6-9,17,18$ and 20-22), worries (items 10-16 and 19, also called the reversed items) and prenatal preparation (items 1 and 2). For each item, there is a single choice to make out of four possibilities from a Likert scale (quite, partly, not so much or not at all). Thus, an average score can be calculated for each dimension: one for the degree of professional support (the lower the score, the higher the support), one for the level of worries (the higher the score, the higher the worries), and one for the effectiveness of the prenatal preparation (the lower the score, the better the prenatal preparation).

The intervention group received a questionnaire to specifically assess their satisfaction with the antenatal classes. It included three assessment statements: (1) "Participating in this session gave me the information I was missing," (2) "I enjoyed being men only," and (3) "After this session, I feel better prepared for the upcoming arrival of my child." Men were asked to respond to four statements according to a Likert scale (not at all, a little, a lot, completely). They could also write commentaries.

\section{Statistical analyses}

Analyses were performed using the STATA software package version 15 (StataCorp, 2017). Continuous variables were checked for impossible values that were re-coded as missing. Furthermore, participants were excluded from the study if they did not complete more than five items on the FTFQ. The final total of participants included in the study and analysis was 173. Categorical variables are reported as number and percentages and continuous data as mean (SD). The two samples were compared with t-tests for normally distributed continuous data and with the Mann-Whitney test for continuous data with a skewed distribution. Chi-squared tests, or Fisher's exact tests when appropriate, were used for categorical variables. In order to test the effect, the difference between the intervention and the non-intervention groups while controlling for the mode of birth, the latter was added as a predictor in an ANOVA conducted for each of the three FTFQ's dimensions. The associations were considered as statistically significant when the p-value was below 0.05 (probability of type I error $=0.05$ ).

\section{Ethical statement}

The questionnaires were anonymous. Only a coding key could link them to the participants' contact information registered in a secure storage space. In accordance with the Swiss federal law on research on human beings, the Geneva Canton Ethics Commission president confirmed that the Ethics Commission did not need to review this project because the aim was outside of the scope of the law. As the scope of 
the Swiss law in the Article 2 of the Human Research Act (HRA) defines, this act applies to research concerning human diseases and concerning the structure and function of the human body (Req-202100629).

\section{Results}

A total of 121 first-time fathers out of 263 ( $46 \%$ response rate) for the non-intervention group completed the FTFQ, and 52 of 103 ( $50 \%$ response rate) for the intervention group.

\section{Demographics and Antenatal Education (Table1)}

There were no significant differences between the intervention group and the non-intervention group on age nor country of origin.

The antenatal education (AE) assessed in this study involved antenatal classes, personal research and Internet searches. In the non-intervention group, $59.6 \%$ of the participants attended a traditional antenatal class with their partners, whereas $100 \%$ of the intervention group had a specifically designed antenatal class for first-time fathers only. Regarding the other means of $A E$, when compared to the non-intervention group, participants in the intervention group felt significantly more prepared from personal research $(61.54 \%$ vs. $30.58 \%-p<0.001)$ and Internet searches $(42.31 \%$ vs. $20.66 \%-p=0.003)$.

\section{Labor and birth (Table 1)}

The use of anesthetics during labor was statistically different with $87.07 \%$ used in the non-intervention group versus $83.67 \%$ in the intervention one $(p=0.028)$.

Regarding the mode of birth, the proportions differed greatly between both groups as shown in table 1 . There were more emergency caesarean sections (EMCS) and less instrumental births and elective caesarean sections (ELCS) in the non-intervention group.

\section{FTFQ}

Table 2 shows the unfavorable answers for the FTFQ. These are the options "somewhat true" and "not true at all" for the normal items, and "partly true" and "completely true" options for the reversed items. Items with unfavorable responses were considered as such when these answers' rate was above $20 \%$ (in bold in Table 2). There were less unfavorable items in the intervention group compared to the nonintervention one, but the differences were not statistically significant. For items 14 and 16, the trend was the opposite.

Feeling well prepared (item 2) was statistically significant between groups, favouring the intervention one.

Factors influencing fathers' experiences of childbirth 
Out of the three FTFQ's dimensions, there was a statistically significant changes in favour of the intervention group for the professional support and prenatal preparation dimensions. As a reminder, for these two, the lower the score, the better the dimension. For the professional support, the scores were 1.98 $(S D=0.52)$ in the non-intervention group and $1.69(S D=0.55)$ in the intervention one $(p=0.001)$; and for the prenatal preparation, the scores were respectively $1.70(S D=0.57)$ and $1.48(S D=0.51)(p=$ 0.019) (Table 3). As the table 1 showed a significant difference between groups for the mode of birth, we adjusted for this variable possibly influencing childbirth experience. An ANOVA revealed that the mode of birth was not a confounding factor on the impact of the intervention on the three dimensions of first-time fathers' experiences.

\section{Feelings of usefulness}

First-time fathers' feelings of usefulness during labor and birth as well as their personal experience of the whole birth process were assessed. A trend towards a better score in the intervention group has been observed, but the differences were not statistically significant. (Table 4)

\section{Satisfaction of specific antenatal classes for men}

During the study period, a total of 21 antenatal classes were offered to pregnant women partners, firsttime fathers-to-be or not. In total, 164 registered, 147 came and 122 responded to the satisfaction questionnaire ( $83 \%$ response rate). Only one was not a first-time father and then excluded from this analysis.

Among them, 95\% attended only one specific antenatal class for men. Their responses to the three questions were as follows:

(1) "Participating in this session gave me the information I was missing": $0 \%$ not at all, $14 / 122$ (12\%) a little, 72/122 (59\%) a lot, 35/122 (29\%) completely

(2) "I enjoyed being male only": 0\% not at all, 6/122 (5\%) little, 61/122 (50\%) a lot, 51/122 (45\%) completely

(3) "After this session, I feel better prepared for the upcoming arrival of my child": $0 \%$ not at all, $28 / 122$ (23\%) a little, 58/122 (48\%) a lot, 33/122 (27\%) completely

In the open comments section, 110/122 (90\%) participants expressed themselves. They emphasized the opportunity to be among men: "This is the only space I know of to discuss pregnancy issues that concern dads." "Open to talk. No filter. No women. Feeling like I'm understood." They appreciated a facilitating educational process: "The few numbers of participants that allows for better interaction." "The organization based on the sharing of tools to help the reflection (pictures, cards)." They also highlighted the solidarity, closeness and sharing: "Sharing concerns." "Closeness." "Taking a break during pregnancy and taking a step back with other future fathers who have the same concerns/problems." 
They suggested some aspects that could be improved, such as teaching skills that are more practical: "A more readable thread on the logistical aspect." "Have a dummy to learn how to hold the baby." They would have like to have had more and longer sessions: "Two hours went by very quickly." "Small break to talk with others."

\section{Discussion}

The need for partner-inclusive intervention strategies and tailored preparation tools for new fathers-to-be have been suggested recently [10,16-18]. In this study, we attempted to evaluate the impact of a specifically designed antenatal class for first-time fathers on their experience of childbirth.

\section{Effect on Labor and Birth}

We observed that the use of anesthetics was statistically reduced in the intervention group. This is in line with the fact that having a trusted birth partner present throughout labor makes a significant difference on the use of epidural, and the safety and wellbeing of women in childbirth [23]. The question in this study was broad and there was no difference made between the different types of pain relief in labor, which first-time fathers could have interpreted as the use of epidural because this is the most common practice in Switzerland. Regardless, a hypothesis in regard to using anesthetics could be that partners felt more prepared and more comfortable supporting their wives, which led them to request less anesthesia.

Regarding the mode of birth, this study shows a significant difference for the mode of birth with a major increase of ELCS in the intervention group. It is difficult to explain this increase because it contrasts with a decrease in the overall caesarean rate in the maternity unit where the study was conducted, from $30 \%$ in 2016 to $24.7 \%$ in 2020. In addition, the study does not specify the reason for ELCS and includes those for maternal request as well as those for medical reasons. This result is surprising because the studied population consisted of first-time fathers, meaning their partners would theoretically more likely be firsttime mothers as well, which would not be in favor of medical reasons explaining ELCS. One hypothesis is that men who enrolled in the specific prenatal classes had partners whose pregnancies were more at risk. We have not been able to verify this hypothesis. However, one of the important results of the study is that the mode of birth does not impact on the effect of specific classes on first-time fathers' experiences of childbirth evaluated through the FTFQ.

\section{Effect on Childbirth Experience}

First-time fathers who attended a specifically dedicated antenatal session felt statistically significantly well prepared [item 2 from the FTFQ: "I felt well prepared."]. The results of the impact of the intervention on the prenatal preparation dimension reinforces this conjecture. We show that the intervention improved the prenatal preparation experience of first-time fathers [ $p=0.02]$. Bradley and Slade [24] found that feeling less prepared was related with anxiety for fathers. Thus, feeling prepared could positively affect fathers' mental health. Baldwin, S. et al [25] also supported that providing fathers with adequate information in the antenatal period to help them better prepare for changes and challenges ahead was 
likely to make their transition to fatherhood more positive and could reduce their stress levels. Furthermore, male involvement interventions also seem to be related to positive outcomes for maternal and newborn health [26]. Further research would be interesting for investigating the effect of these specific antenatal sessions on fathers' mental health and indirectly on maternal and newborn health.

Our study also showed a positive statistically significant difference of the effect of specifically designed antenatal sessions for first-time fathers on the professional support dimension. Following the validation of the FTFQ in French, the results from the non-intervention group were shown to the maternity healthcare professionals for them to realize the need of supporting partners. It is possible that the teams changed their behavior in favor of better support to partners, which could explain these results. However, it could also infer to the fact that first-time fathers felt well prepared and therefore more as part of a team $[10,26]$.

This study did not show any differences regarding the effect of the intervention on the worries dimension. It is surprising to note that the fact of feeling well prepared and well supported by professionals did not affect the worries feelings. Childbirth is a moment associated with high uncertainty, which does not depend on the antenatal classes or the support the families receive [27]. A new questioning arises from these results, wondering if it is possible to reduce first-time fathers' level of worries during childbirth.

Regarding of the other items of the FTFQ, although the results are not statistically significant, all the unfavorable responses were reduced in the intervention group. Only two items were higher ["I was worried that I wouldn't be able to provide support" and "I was worried about how I would react."]. This could be related to the mode of delivery because the ELCS rate tripled in this group $[28,29]$.

Finally, we measured a benefit while $95 \%$ of participants attended only one specific antenatal classes. It is likely that the results would be even better after attending several sessions.

\section{Satisfaction of the antenatal classes for partner}

All fathers had access to extra antenatal education along with the antenatal classes. However, there were statistical differences for extra antenatal education regarding personal research and education from the Internet, which could relate to how the intervention group had greater proportions of participants with higher education [30].

The results of the satisfaction survey of fathers after the antenatal classes were very positive. In addition to feeling better prepared for their child's arrival, they appreciated having a space specifically dedicated to men. The experience reported in terms of sharing and solidarity between them seems to have been strong from a human point of view. In their review of mental health problems in fathers following the birth of a child, Bradley and Slade [24] reported, among other factors, the negative influence of the father's psychological distress during pregnancy and of the poor social adjustment during their partner's pregnancy. Involving men during pregnancy is often recommended to improve maternal and newborn health [26]. There are high societal expectations on fathers' attitude during pregnancy, delivery and postpartum, but with very little support for themselves. However, scientific research in the last decade has 
highlighted their own vulnerability during their identity transition to parenthood. The antenatal classes specifically design for partners are an opportunity for professionals and partner pairs to offer real support to the fatherhood process.

\section{Strengths And Limitations}

To our knowledge, this is the first study that investigates the effect of specifically designed antenatal classes for first-time fathers on their experience of childbirth. The tool used for evaluating the childbirth experience has been validated for its psychometric properties [20].

The 6-year gap between the two groups, the difference in the sample sizes of the two groups as well as the fact that it was a retrospective study represent this study's limitations. Indeed, the practices, protocols and recommendations have evolved in this period and could explain some of the differences found. Moreover, some questions, such as the use of anesthesia, were too broad and the analysis was not optimum, although the difference was significant between the control and intervention groups.

It would be interesting to conduct further research about the different uses of pain relief options and the partner's involvement in this choice, depending on if they had attended a first-time father's specific antenatal session. Further research with a bigger sample, randomized and prospective could also be interesting to investigate the trend found in the unfavorable answers as part of the FTFQ, looking at the improvement of first-time fathers' experience of childbirth and their feelings of usefulness in labor and during birth if they attended specifically designed antenatal classes.

\section{Conclusions}

This study has shown that attending specific antenatal classes for first-time fathers improves their experience of childbirth. Implementing these as a routine in the antenatal period would be a good way to involve first-time fathers more in pregnancy care and to support them in their journey to fatherhood. Their experience reported in terms of sharing and solidarity between men seems to be meaningful. Offering them a specifically dedicated space for expression is a simple way to support their transition to fatherhood. To broaden the question, another source of investigation could be if the antenatal sessions being held solely by male midwives are an important factor, or if is it the fact that first-time fathers meet with their peers that makes the difference. We also suggest extending and evaluating access to these preparations to non-gendered communities.

\section{Declarations}

\section{Conflict of interest}

The authors declare that they have no conflict of interest.

\section{Authorship contribution statement}


Marie-Julia Guittier: conception and design, acquisition of data, analysis and writing. Isabelle Cornet: analysis, interpretation of data and writing. Begoña Martinez de Tejada: interpretation of data and writing. All authors approved the final version to be submitted.

\section{Fundings}

This research was supported by the School of Health Sciences Geneva; HES-SO University of Applied Sciences and Arts, Western Switzerland; the Obstetrics Division of the University hospitals of Geneva and the Foundation for the Research in Perinatal (www.frep-swiss.net).

\section{Acknowledgements}

We thank all the men who participated in this study and professionals in the maternity unit of the Geneva university hospitals who facilitated the research.

\section{References}

1. Genesoni L, Tallandini MA. Men's Psychological Transition to Fatherhood: An Analysis of the Literature, 1989-2008. Data Collect. 2009;13.

2. Håland K, Lundgren I, Lidén E, Eri TS. Fathers' experiences of being in change during pregnancy and early parenthood in a context of intimate partner violence. Int J Qual Stud Health Well-Being. janv 2016;11(1]:30935.

3. Condon J. What about dad? Psychosocial and mental health issues for new fathers. Aust Fam Physician. sept 2006;35(9]:690-2.

4. World Health Organization. WHO recommendations on health promotion interventions for maternal and newborn health (Internet]. WHO Library Cataloguing-in-Publication Data; 2015. Disponible sur: https://www.ncbi.nlm.nih.gov/books/NBK304983/pdf/Bookshelf_NBK304983.pdf

5. Johansson M, Fenwick J, Premberg å. A meta-synthesis of fathers $\square$ experiences of their partner $\square s$ labour and the birth of their baby. Midwifery. janv 2015;31(1]:9-18.

6. van Vulpen $M$, Heideveld-Gerritsen $M$, van Dillen J, Oude Maatman S, Ockhuijsen $H$, van den Hoogen $A$. First-time fathers' experiences and needs during childbirth: A systematic review. Midwifery. mars 2021;94:102921.

7. Inglis $C$, Sharman R, Reed R. Paternal mental health following perceived traumatic childbirth. Midwifery. oct 2016;41:125-31.

8. Ledenfors A, Berterö C. First-time fathers' experiences of normal childbirth. Midwifery. sept 2016;40:26-31. 
9. Vallin $\mathrm{E}$, Nestander $\mathrm{H}$, Wells MB. A literature review and meta-ethnography of fathers' psychological health and received social support during unpredictable complicated childbirths. Midwifery. janv 2019;68:48-55.

10. Baldwin S, Malone M, Sandall J, Bick D. Mental health and wellbeing during the transition to fatherhood: A systematic review of first time fathers' experiences. JBI Database Syst Rev Implement Rep. nov 2018;16:2118-91.

11. Madsen SA, Munck H. (Fathers' presence at deliveries in Denmark.]. Sante Ment Que. 2001;26(1]:27-38.

12. O'Brien AP, McNeil KA, Fletcher R, Conrad A, Wilson AJ, Jones D, et al. New Fathers' Perinatal Depression and Anxiety-Treatment Options: An Integrative Review. Am J Mens Health. juill 2017;11(4]:863-76.

13. Paulson JF, Bazemore SD. Prenatal and postpartum depression in fathers and its association with maternal depression: A meta-analysis. J Am Med Assoc. 2010;303:1961-9.

14. Howarth AM, Scott KT, Swain NR. First-time fathers' perception of their childbirth experiences. J Health Psychol. 2017;24:929-40.

15. Vehviläinen-Julkunen K, Liukkonen A. Fathers' experiences of childbirth. Midwifery. mars 1998;14(1]:10-7.

16. Hughes C, Foley S, Devine RT, Ribner A, Kyriakou L, Boddington L, et al. Worrying in the wings? Negative emotional birth memories in mothers and fathers show similar associations with perinatal mood disturbance and delivery mode. Arch Womens Ment Health. juin 2020;23(3]:371-7.

17. Berlin A, Rosander M, Frykedal KF, Törnkvist L, Barimani M. Fatherhood group sessions: A descriptive and summative qualitative study. Nurs Health Sci. déc 2020;22(4]:1094-102.

18. Henry JB, Julion WA, Bounds DT, Sumo J. Fatherhood Matters: An Integrative Review of Fatherhood Intervention Research. J Sch Nurs Off Publ Natl Assoc Sch Nurses. févr 2020;36(1]:19-32.

19. Poh HL, Koh SSL, Seow HCL, He H-G. First-time fathers' experiences and needs during pregnancy and childbirth: A descriptive qualitative study. Midwifery. juin 2014;30(6]:779-87.

20. Capponi I, Carquillat P, Premberg $\AA$, Vendittelli F, Guittier M-J. Vécu de l'accouchement par les pères: Traduction et validation transculturelle du First-Time Father Questionnaire sur un échantillon francophone. Gynécologie Obstétrique Fertil. sept 2016;44(9]:480-6.

21. Premberg $\AA$, Taft C, Hellström A-L, Berg M. Father for the first time - development and validation of a questionnaire to assess fathers' experiences of first childbirth (FTFQ]. BMC Pregnancy Childbirth 
(Internet]. déc 2012 (cité 12 févr 2019];12(1]. Disponible sur:

https://bmcpregnancychildbirth.biomedcentral.com/articles/10.1186/1471-2393-12-35

22. Premberg $\AA$, Taft C, Hellström A-L, Berg M. Father for the first time - development and validation of a questionnaire to assess fathers' experiences of first childbirth (FTFQ]. BMC Pregnancy Childbirth (Internet]. déc 2012 (cité 12 févr 2019];12(1]. Disponible sur: https://bmcpregnancychildbirth.biomedcentral.com/articles/10.1186/1471-2393-12-35

23. Bohren MA, Hofmeyr GJ, Sakala C, Fukuzawa RK, Cuthbert A. Continuous support for women during childbirth. Cochrane Pregnancy and Childbirth Group, éditeur. Cochrane Database Syst Rev (Internet]. 6 juill 2017 (cité 22 févr 2019]; Disponible sur: http://doi.wiley.com/10.1002/14651858.CD003766.pub6

24. Bradley R, Slade P. A review of mental health problems in fathers following the birth of a child. J Reprod Infant Psychol. févr 2011;29(1]:19-42.

25. Baldwin S, Malone M, Sandall J, Bick D. Mental health and wellbeing during the transition to fatherhood: A systematic review of first time fathers' experiences. JBI Database Syst Rev Implement Rep. nov 2018;16:2118-91.

26. Tokhi M, Comrie-Thomson L, Davis J, Portela A, Chersich M, Luchters S. Involving men to improve maternal and newborn health: A systematic review of the effectiveness of interventions. van Wouwe JP, éditeur. PLOS ONE. 25 janv 2018;13(1]:e0191620.

27. Philpott LF, Leahy-Warren P, FitzGerald S, Savage E. Stress in fathers in the perinatal period: A systematic review. Midwifery. déc 2017;55:113-27.

28. Hasman K, Kjaergaard H, Esbensen BA. Fathers' experience of childbirth when non-progressive labour occurs and augmentation is established. A qualitative study. Sex Reprod Healthc. juin 2014;5(2]:69-73.

29. Yokote N. Fathers' feelings and thoughts when their partners require an emergency cesarean section: Impact of the need for surgery: Fathers' feelings about emergency cesarians. Jpn J Nurs Sci. 7 déc 2007;4(2]:103-10.

30. Tomintz MN, Clarke GP, Rigby JE, Green JM. Optimising the location of antenatal classes. Midwifery. janv 2013;29(1]:33-43.

\section{Tables}

Table 1: Demographics, antenatal education and birth data 


\begin{tabular}{|c|c|c|c|}
\hline & $\begin{array}{l}\text { Non-Intervention } \\
\mathrm{N}=121\end{array}$ & $\begin{array}{l}\text { Intervention } \\
\mathrm{N}=52\end{array}$ & \\
\hline Variable & Mean (SD) & Mean (SD) & $\mathrm{P}$ (t-test) \\
\hline Age & $33.5(5.12)$ & $35.08(5.46)$ & 0.0768 \\
\hline Variables & $\mathrm{n}(\%)$ & $\mathrm{n}(\%)$ & $\begin{array}{l}\text { P } \\
\text { (chi-square test) }\end{array}$ \\
\hline Country of birth: & & & 0.958 \\
\hline Switzerland & 75 (64.66) & $33(63.46)$ & \\
\hline Europe & $23(19.83)$ & $10(19.23)$ & \\
\hline Other & $18(15.52)$ & $9(17.31)$ & \\
\hline Levels of Education: & & & 0.082 \\
\hline Minimum Education & $22(18.80)$ & $8(15.69)$ & \\
\hline High School / A Levels & $27(23.08)$ & $5(9.80)$ & \\
\hline University & $68(58.12)$ & $38(74.51)$ & \\
\hline Onset of Labor: & & & 0.243 \\
\hline Spontaneous & $58(52.73)$ & $20(42.55)$ & \\
\hline Induced & $52(47.27)$ & $27(57.45)$ & \\
\hline Anesthesia: & $101(87.07)$ & $41(83.67)$ & 0.028 \\
\hline Mode of Birth: & & & 0.034 \\
\hline SVB & $63(52.07)$ & $19(36.54)$ & \\
\hline Vacuum/Forceps & $23(19.01)$ & $15(28.85)$ & \\
\hline ELCS & $6(4.96)$ & $8(15.38)$ & \\
\hline EMCS & $29(23.97)$ & $10(19.23)$ & \\
\hline AE: Personal Research & $37(30.58)$ & $32(61.54)$ & $<0.001$ \\
\hline AE: Internet & $25(20.66)$ & $22(42.31)$ & 0.003 \\
\hline
\end{tabular}




$\begin{array}{llll}\text { AE: Friends and Family } & 57(47.11) & 26(50.00) & 0.727\end{array}$

Table 2: Descriptive analysis of the unfavorable responses for all the items in the FTFQ

${ }^{1}$ fisher exact test

${ }^{2}$ chi-square test

* These items are part of Premberg et al.'s FTFQ in Swedish. However, they were not validated for the French version of the test; therefore, their means were not calculated.

Items in bold are the unfavorable answers.

Table 3: Means for each dimension from the FTFQ_

\begin{tabular}{|lllll|}
\hline & $\begin{array}{l}\text { Non-Intervention } \\
\mathrm{N}=121\end{array}$ & $\begin{array}{l}\text { Intervention } \\
\mathrm{N}=52\end{array}$ & $\begin{array}{l}\text { Adjusted for } \\
\text { mode of birth }\end{array}$ \\
\hline Factors & Mean (SD) & Mean (SD) & P (t-test) & P (Anova) \\
\hline Professional Support & $1.98(0.52)$ & $1.69(0.55)$ & $\mathbf{0 . 0 0 1 3}$ & $\mathbf{0 . 0 0 5}$ \\
\hline Worries & $2.32(0.74)$ & $2.26(0.73)$ & 0.6072 & 0.480 \\
\hline Prenatal Preparation & $1.70(0.57)$ & $1.48(0.51)$ & $\mathbf{0 . 0 1 9 2}$ & $\mathbf{0 . 0 3 9}$ \\
\hline
\end{tabular}

Table 4: Feelings of Usefulness and Overall Experience

\begin{tabular}{|llll|}
\hline & Non-Intervention & Intervention & \\
& $\mathrm{N}=\mathbf{1 2 1}$ & $\mathrm{N}=\mathbf{5 2}$ & \\
& & & \\
\hline Variables & Mean (SD) & Mean (SD) & P (Mann-Whitney) \\
\hline Usefulness in Labor & $7.10(2.57)$ & $7.57(2.39)$ & 0.230 \\
\hline Usefulness during Birth & $6.81(3.00)$ & $7.39(2.45)$ & 0.455 \\
\hline Overall Experience & $8.11(2.59)$ & $8.63(1.63)$ & 0.830 \\
\hline
\end{tabular}




\section{Items}

\section{Non-}

Intervention

$N=121$
Intervention

$P$

$N=52$

n (\%)

$\mathrm{n}(\%)$

Item 1: I felt well informed.

$9(7.44)$

$3(5.77)$

$1.000^{1}$

Item 2: I felt well prepared.

$16(13.22)$

$1(1.92)$

$0.024^{1}$

Item 3: We were admitted to the maternity unit we had chosen.

Item 4: I felt welcome when I called the maternity unit.

Item 5: I was treated well on arrival at the maternity unit.

Item 6: I felt the staff gave me positive attention.

Item 7: I was given enough information.

Item 8: I was given guidance on how to support my wife/girlfriend.

Item 9: There was some information I lacked.

Item 10: There were situations I would rather not have gone through.

Item 11: I was worried about my wife/girlfriend.

Item 12: I was worried about the baby.

Item 13: I was worried that something would go wrong.

Item 14: I was worried that I would not be able to provide support.

Item 15: I was worried about the unknown.

Item 16: I was worried about how I would react.

Item 17: I felt that the midwives and other staff were interested in how I felt.

Item 18: The staff offered to support my wife/girlfriend so that I could take a break.

Item 19: There were things that frightened me during childbirth.
$4(7.69)$

$1.000 *$

$1(0.83)$

$1(1.92)$

$0.512^{\star}$

$2(1.65)$

$2(3.85)$

$0.585^{\star}$

$15(12.40)$

$4(7.69)$

$0.437^{1}$

$13(10.74)$

$2(3.85)$

$28(23.14) \quad 9(17.31)$

$0.237^{1}$

$41(33.88) \quad 12(23.08)$

$0.157^{2}$

$30(24.79)$

$13(25.00)$

$0.977^{2}$

$80(66.12)$

33 (63.46)

$0.737^{2}$

$73(60.33) \quad 28(53.85) \quad 0.428^{2}$

81 (66.94) $\quad 28(53.85) \quad 0.102^{2}$

$54(44.63) \quad 26(50.00) \quad 0.516^{2}$

$66(54.55) \quad 22(42.31) \quad 0.140^{2}$

$34(28.10) \quad 17(32.69) \quad 0.544^{2}$

31 (25.62) $\quad 10(19.23) \quad 0.365^{2}$

$78(64.46) \quad 30(57.69) \quad 0.399^{2}$

$19(15.70) \quad 8(15.38)$

$0.958^{2}$
$9(7.44)$ 
Item 20: I was hugged and comforted when I was upset.

Item 21: I was shown how to hold the baby.

Item 22: I was encouraged to hold the baby.
$21(17.36)$

$37(30.58)$

$25(20.66)$
$9(17.31)$

$10(19.23)$

$0.124^{2}$

$5(9.62)$ $0.078^{2}$ 